\title{
ABORDAJE DE LOS PROCESOS DE LECTURA Y ESCRITURA EN UNA ESCUELA: PROPUESTA PEDAGÓGICA EN Y PARA LA DIVERSIDAD
}

\author{
M.Ed. Silvia Elena León Muñoz ${ }^{1}$
}

\section{Resumen}

El presente trabajo surgió como producto de una investigación cuyo objetivo era desarrollar las habilidades y destrezas para la adquisición de los procesos de lectura y escritura, partiendo de los antecedentes lingüísticos de la niña y el niño, para lograr niveles superiores, desde una perspectiva de la educación en y para la diversidad.

La literatura consultada demuestra la importancia de la perspectiva de la diversidad en congruencia con el desarrollo de los procesos de lectura y escritura, al entender las múltiples características que poseen el niño y la niña preescolares para construir las diversas habilidades $y$ destrezas. Asimismo, permite un nuevo cambio en la sociedad y para ello, el currículo de una escuela en y para la diversidad propone un acceso real de parte de los estudiantes, apertura y flexibilidad.
Además, se desarrolla en los párvulos el valor de lo diferente como una riqueza para la convivencia.

Se describen en términos generales cinco talleres que proponen el desarrollo de las habilidades y destrezas de la lectura y la escritura; a la vez, se retoma en todos el valor de promover en las niñas y los niños la convivencia en la diversidad que cada uno posee, concibiendo la diversidad como una riqueza para la interacción.

\section{Palabras claves}

Educación preescolar, diversidad, procesos de lectura y escritura.

\section{Abstract}

This article is the product of research designed to develop abilities and skills required to acquire reading and writing processes. It starts from the linguistic

1 Máster en Pedagogia con énfasis en Diversidad de los Procesos Pedagógicos. Licenciatura en Pedagogia con énfasis en Educación Preescolar. 
background of the child, trying to develop superior levels from the perspective of education for the diversity.

The literature review showed the significance of a perspective "in and for the diversity" in the development of reading and writing processes, by understanding multiple characteristics of pre-schooled children implicated in the construction of different abilities and skills. Considering diversity allows social changes, consequently, school curriculum for the diversity provides real access for students, as well as flexibility. Additionally, children are taught to value diversity as way of enhance interpersonal coexistence.

Five workshops are proposed to develop reading and writing abilities, taking into account the value of diverse interactions.

\section{Key words}

Reading and writing processes, education, diversity, workshops, students.

\section{Introducción}

$\mathrm{E}$ desarrollo integral del niño y la niña preescolares parte de múltiples vivencias y deseos por aprender. Un aspecto de gran importancia en el infante es el desarrollo del lenguaje, el cual le permite comunicarse desde diversas formas, para expresar así sus necesidades, intereses, sentimientos y, por ende, sus pensamientos; para ello, recurre a diferentes códigos. En los primeros años de vida, el niño y la niña logran desarrollar su aparato fonoarticulador, para dar paso a los procesos de la lectura y la escritura.

Danoff, Breitbart y Barr (1996) mencionan que la lectura, por su parte, implica un proceso que pone en juego diversas habilidades de identificación, de comprensión de las palabras y, posteriormente, la lectura con un fin específico, desarrolla contenidos escritos como fuentes de referencia (p. 127). La escritura como tal, requiere de habilidades y destrezas de coordinación motora, que implican el control de los músculos en su totalidad, para lo cual el niño y la niña necesitan de actividades motrices como saltar, brincar, coser, pintar, amasar, dibujar, construir con cubos, entre otras, esenciales en su desarrollo.

Los procesos de lectura y escritura se desarrollan de forma muy diversa en los niños y las niñas, debido a variantes en su desarrollo, deseos e intereses; por lo tanto, al ser un proceso que depende de cada uno, es conveniente que exista una concientización en las docentes de la Educación Preescolar para que atiendan a aquellos que desean iniciar estos procesos, sin segregar a los que aún no tienen el interés. Esto propicia una aceptación de la diversidad en los ritmos y estilos de aprendizaje del infante. 
Por lo anterior, es importante elaborar una propuesta pedagógica para el abordaje de los procesos de lectura y escritura, dentro una perspectiva de la diversidad en los procesos de enseñanza y aprendizaje, con el fin de cumplir el objetivo de atender a los niños y las niñas en sus deseos, intereses y necesidades por aprender un código convencional.

Si bien es cierto que el Ciclo de Preescolar no tiene como objetivo que la niña y el niño aprendan a leer y escribir, no se deja de atender a aquellos que tienen la inquietud, las destrezas y las habilidades necesarias, porque ante todo es una necesidad e interés del infante. Tal situación acoge una de las características de la declaración de los derechos humanos, según la debida educación y estimulación temprana del sujeto. Con esto, se recalca la importancia de que si un niño o niña no posee el interés en esta edad preescolar, no se debe forzar lo que aún no es de trascendencia para el individuo. Como todo proceso, necesita de afianzamiento y voluntad del individuo para salir adelante cuando esté preparado; forzarlo sería inconveniente para su desarrollo integral.

La temática que aborda este trabajo surge a partir de la experiencia que radica en el ejercicio de la docencia en los Ciclos de Transición y Materno Infantil, donde las vivencias pedagógicas permiten conocer los procesos de la lectura y la escritura de niños y niñas con edades preescolares, convirtiéndose en un tema de gran importancia la forma en cómo se pueden desarrollar esos procesos.

Cid (1997) permite hacer la relación con tales procesos y una posible condición de pobreza, al referirse a la poca estimulación que existe en los hogares pobres, y su implicación en el desarrollo de los infantes. Entonces, se da una gran brecha entre quienes poseen una estimulación y quienes no. Asimismo, se presentan situaciones donde los progenitores no han sido alfabetizados y este es un factor que, probablemente, incide en el proceso de aprendizaje.

Cuando las niñas y los niños llegan a las aulas, es necesario realizar un diagnóstico, que brinde un panorama de lo que saben y conocen, y a partir de ahí se elaboran los planes necesarios para buscar un mejor acercamiento a los procesos, que respondan a sus particularidades, y brindar la estimulación necesaria.

Se deben considerar, por lo tanto, las diversidades personales o individuales, las cuales comprenden el nivel de desarrollo, la edad, el sexo, el género, los aspectos físicos (salud, posibles discapacidades, apariencia), rendimiento académico, motivación para aprender, estilos y ritmos de 
aprendizaje, estimulación, acceso a recursos, lenguaje, comunicación, conocimientos previos, expectativas, intereses, desarrollo socioemocional-madurez, personalidad, conducta y valores. Del mismo modo, han de considerarse las diversidades contextuales como el tipo de educación, el entomo físico y ecológico, la nacionalidad, nivel socioeconómico, la cultura y la familia que cada estudiante presenta como punto de inicio antes de elaborar las estrategias metodológicas.

La posición y perspectiva sobre la temática de los procesos de lectura y escritura deben circunscribirse a una visión de la diversidad, entendida como una combinación de características que poseen los seres humanos, las cuales, por su naturaleza, tienen un aspecto determinado, que son sus diferencias individuales, tanto en lo social, cultural, sexual, como de aprendizaje.

Esta diversidad es más notable en cada grupo de estudiantes en niveles determinados, y el nivel preescolar no es la excepción. Más bien, se convierte en un nivel clave para conocer a cada individuo y lograr así un mejor acercamiento a los procesos cognitivos, sin lastimar su desarrollo integral y considerándolo como el protagonista de este proceso.

El desarrollo de la lectura y la escritura en la edad preescolar puede ser un primer acercamiento a la metacognición, porque al saber el niño y la niña lo que hacen, es decir, leer y escribir, que sería una decodificación del contexto, estarían haciendo un proceso de reflexión, que les permitiría comprender el significado del lenguaje.

Para la operacionalización de la propuesta para el abordaje de los procesos de lectura y escritura, se deben tener claros ciertos elementos, con el fin de no alejarse de la idea de la educación en y para la diversidad. El primer elemento que fortalece esta propuesta es que todos los estudiantes tienen las mismas oportunidades para acceder al aprendizaje; por lo tanto, se habla de inclusión, contemplando a los niños y las niñas como sujetos independientemente de sus condiciones fǐsicas, intelectuales, emocionales, lingüísticas u otros niños o niñas discapacitados, bien dotados, de la calle, que trabajan o de pueblos remotos. Este planteamiento tiene relación con los derechos humanos y con la oportunidad de una calidad de vida, que satisfaga lo cognitivo, lo social y lo personal.

El segundo elemento enfatiza en el principio de la atención a la diversidad, ya que la propuesta ofrece una serie de actividades, mediante talleres, para que los niños y las niñas participen, experimenten y vivencien, de acuerdo con sus posibilidades, potencialidades y sus características personales. 
Un tercer elemento de la propuesta se refiere al marco de esta con un enfoque de dinamismo. Las niñas y los niños pueden elegir democráticamente lo que realizarán, tendrán apoyos y oportunidades, flexibilidad, coevaluación y autocriticismo para valorar el producto logrado.

En síntesis, esta propuesta es una alternativa curricular con estrategias que posibilitan el acercamiento a la lectura y la escritura de los niños y las niñas preescolares, tomando en cuenta sus características personales y culturales, es decir, aceptando su diversidad; su fin radica en disminuir la cantidad de niños y niñas que no pueden acceder a estos procesos en forma asertiva.

La propuesta será un instrumento para aquellos docentes que identifiquen en sus intervenciones pedagógicas el interés de sus estudiantes en los procesos de lectura y escritura, desde la diversidad que representa cada uno; a la vez, se busca la no segregación de los estudiantes que aún por su edad no sienten el deseo e interés por estos procesos.

En resumen, el objetivo es que la niña y el niño desarrollen sus habilidades y destrezas para la adquisición de los procesos de lectura y escritura, partiendo de los antecedentes lingüísticos que poseen, logrando así niveles superiores.

\section{Selección del contexto institucional}

El abordaje de los procesos de la lectura y la escritura desde un paradigma de la diversidad, requiere de una propuesta que logre atender las necesidades e intereses, partiendo de un diagnóstico que brinde las aportaciones necesarias para la elaboración de los objetivos y estrategias de aprendizajes, que puedan dar respuesta a las necesidades de un grupo de infantes.

Por ello, esta Propuesta Pedagógica parte, como se mencionó anteriormente, del diagnóstico realizado durante el año 2003 con estudiantes del nivel de kínder. La implementación de la Propuesta Pedagógica se diseñó para una institución ${ }^{2}$ en particular y abre la posibilidad para que también sea puesta en marcha en cualquier otro centro educativo.

\section{Identificación de la situación problema}

El diagnóstico mencionado fue aplicado en el año 2003, a un grupo de niños y niñas del nivel de kínder (o materno infantil), y se desarrolló mediante

2 La institución educativa es de carácter privado y está ubicada en la provincià de San Josć, en el cantón de Escazí, en el Circuito 08 de la Regional de San Josć. 
un Taller de Lectura Creativa, cuyo propósito u objetivo fue conocer las diferentes formas en las que les gusta expresar su conocimiento. El proceso se inscribió desde una perspectiva del paradigma naturalista, que se centra en el estudio de las acciones del ser humano en la vida social. Se utilizaron dos instrumentos que permitieron recolectar la información: el primero fue la actividad recreativa, es decir, el Taller de Lectura Creativa. El segundo fue una Guía de Observación, donde se anotaba la información más relevante durante el proceso.

En este grupo de kínder, la diversidad es notable y se comprobó a partir del Taller de Lectura Creativa; ante todo, es necesario indicar que las actividades fueron propuestas por los niños y las niñas; en otras palabras, la guía surgió de la experiencia, intereses y necesidades de los estudiantes, quienes además de participar en lo que más les atrae, construyen al mismo tiempo diferentes conocimientos, tanto conceptuales, como actitudinales que son de gran importancia para mantener mejores interacciones con el medio y quienes lo conforman.

Finalizado el diagnóstico, se puede afirmar que la diversidad es parte de la rutina diaria del ser humano; cada persona posee diferentes gustos, estilos de aprendizaje, conocimientos previos y ello configura una gama de diferencias que a la vez las enriquece en el diario vivir.

El nivel preescolar es fundamental, pues es un espacio de socialización; hay que tener en claro que su función es fomentar en el sujeto las actitudes para saber qué quiere y lo que es capaz de realizar; asimismo, le permite desarrollar una serie de competencias mediante estrategias tanto individuales como colectivas, que fomentan un desarrollo integral, una transformación y aceptación de la diversidad; como lo dice los autores Devalle y Vega (1999):

...un sujeto arquitecto de su propio destino, supone el dominio del conocimiento racional y no sólo la adhesión a ciertos valores políticos-ideológicos específicos. El que sabe más tiene más posibilidades de lograr su propia autorrealización. De allí que la socialización creciente del conocimiento tenga potencialidades profundamente democratizadoras. Por lo tanto, es conveniente plantear la complejidad de la identidad del sujeto en el sentido de su construcción permanente (p. 124).

Si bien se habla en este nuevo siglo del cambio, el Sistema Educativo no está al margen de ello y como lo menciona la literatura, es necesario inculcar en los individuos desde pequeños una actitud de respeto por la diferencia y 
visualizarla, al mismo tiempo, como una gran riqueza para aprender y convivir con todos los sujetos del mundo, sin caer en la otredad. La otredad puede ser vista como lo diferente, desde la semántica, el término indica diversidad. De lo anterior, surge la necesidad de una atención a la diversidad, donde los individuos sean considerados como tales, sin querer unirlos en grupos homogéneos. Es necesario, entonces, promover la perspectiva ética, que respete los Derechos Humanos en su totalidad, basándose una vez más en los valores del respeto, la justicia, la libertad para elegir, la igualdad entre todos sin limitaciones y la solidaridad entre todos y todas.

La diversidad escolar se promueve en la convivencia de cada uno de los implicados en el proceso de enseñanza y aprendizaje. Así se responde a lo que Aguilar (2000) propone como razones para atender la diversidad, en donde el individuo posee un proceso de aprendizaje propio e individual, pero - que necesita de la interacción con el medio.

Cada persona aprende de diversas formas, porque tal y como lo explican las teorías de Vygotsky y Piaget, el desarrollo es diferente en los sujetos y la interacción social, en la medida en que el niño y la niña crecen, llegan a asumir sus roles en la construcción del conocimiento como en la visión del entorno; de ello dependen los procesos de desarrollo y de cambios en sus niveles de madurez, crecimiento y experiencias de aprendizaje; por lo tanto, se encuentra un sujeto con conocimientos previos. Los conocimientos previos son una herramienta para la docente; de ellos se debe arrancar para proponer las actividades significativas y la motivación del docente es fundamental, para que se dé un proceso de aprender a aprender.

\section{La pluralidad en el contexto moderno y sus implicaciones para el desa- rrollo de la lectura y la escritura del niño y la niña preescolares}

La modernidad de este nuevo siglo, se caracteriza por el constante avance en las diversas esferas del entorno social y, por ende, el ser humano inmerso en ese contexto, ha ido desarrollando sus habilidades, para responder a las demandas del mundo actual. El desarrollar el gusto por la lectura y la escritura, en el niño y la niña, es una ventaja, porque les proporciona una apertura a la reflexión; por ello, los procesos de alfabetización deben desarrollar una actitud crítica en los discentes desde las edades más tempranas.

Sin embargo, en la actualidad, tal y como lo señala Giroux (1990), "la cultura visual amenaza la autorrểexión y el pensamiento crítico” (p. 132), mediante el abuso de uso de la televisión, la radio y otros medios de comunicación 
masiva, se ofrece una constante "manipulación y control social" (p. 127), llegando así, a una dimensión de reducción del pensamiento en el ser humano, porque la cultura visual, provoca la poca necesidad de reflexionar sobre lo que pasa.

Esta situación obedece a una herramienta de control por parte del sector de poder. Para enfrentar esta situación, que genera desventajas, es indispensable inculcar en el niño y la niña el gusto por la lectura y la escritura, porque "Al leer, uno dispone de más tiempo para pararse y reflexionar sobre lo que está escrito. Con la palabra escrita es posible evaluar más rigurosamente la validez del valor de verdad de un determinado argumento" (Giroux, 1990, p. 128).

Las sociedades poseen sus propias características, diferencias y diversidades. Cuando se hace referencia a las diferentes formas de vivenciar la existencia del ser humano, es indispensable conceptuar el término de diversidad. Por lo tanto,

Diversidad es una condición de la vida en comunidad, cuyos procesos vitales se relacionan entre sí y se desarrollan en función de los factores culturales de un mismo ambiente. Donde, tanto relación como variedad, aseguran y potencian la vida de todos los habitantes de esa comunidad (Meléndez, 2002, p. 28).

Por ende, se establecen las diferencias propias de cada uno de sus grupos, etnias y habitantes en general, en relación con la visión de la realidad; entonces, surgen múltiples percepciones de lo que ocurre. Bruner (citado por Devalle y Vega, 1999) menciona que:

Conocemos el mundo de diferentes maneras, desde diferentes actitudes y cada una de las maneras en que lo conocemos produce diferentes estructuras o representaciones $o$, de hecho, realidades tanto el mirar como el escuchar están conformadas por expectativas, la actitud y la intención (p. 52).

Tomando como punto de partida la perspectiva anterior, se hace implícita la concepción de una diferencia, que existe en forma activa y perpetua, un concepto que engloba mucho significado, que permite a la vez conocer $y$, sobre todo, entender lo que sucede en este mundo. "La palabra diferencia podría llegar a sugerir cierto parámetro de contraste, cierto modelo de referencia y hasta excelencia" (Devalle y Vega, 1999, p. 39). En la sociedad 
mundial, se logra denotar la multiplicidad de prácticas, formas de vivenciar las situaciones cotidianas; por lo tanto, no existe una única forma de describir esas realidades (como respuesta a determinados propósitos); esta pluralidad de realidades se identifican como diversidades, por ser "considerada como valor implica orientar la educación hacia los principios de igualdad, justicia y libertad, y todo ello para establecer un compromiso permanente con las culturas y los grupos minoritarios" (1999, p. 39).

La diferencia y diversidad no significan lo mismo, cada término posee su contenido; pero entre ellos surge una relación. El anterior planteamiento es resumido de la siguiente forma por las autoras citadas:

Los seres humanos somos diferentes unos de otros y nuestras diferencias se expresan en diversas formas de sentir e interpretar el mundo que cada uno consiruye dentro de un marco histórico social y cultural en el que se desarrolla su existencia (p. 40).

\section{La educación para la diversidad y el contexto educativo desde una visión macro de la sociedad}

La educación en y para la diversidad posee sus fundamentos, visiones y posturas, lo cual se reafirma en el cambio y apertura de las concepciones; al considerar la diversidad como un valor positivo, surge la necesidad de atender las distintas culturas que se integran en el Sistema Educativo; y la función de la escuela es

...dar una respuesta a todos los niños que acuden a ella, no lo puede hacer desde concepciones ni desde prejuicios patológicos y perversos sobre la diversidad, sino desde la consideración de que ser diverso es un elemento de valor y un referente positivo para cambiar la escuela. Todo ello permitirá que los alumnos vayan adquiriendo una cultura distinta al vivir las diferencias entre las personas como algo valioso, solidario y democrático... (López, citado por Devalle y Vega, 1999, p. 39).

La atención a la diversidad parte de un nuevo cambio de la sociedad y, por ende, del currículo que fundamenta el proceso de enseñanza y aprendizaje. Un currículo para una escuela en y para la diversidad tiene una serie de características para que cumpla cabalmente sus objetivos, las cuales se basan en el acceso real al conocimiento de los y las estudiantes; en corisecuencia, debe 
ser abierto, atractivo y flexible en su propuesta para el proceso de enseñanza (Devalle y Vega, 1999, p. 171). Será un currículo alternativo que vivencie implícitamente los valores, la toma de decisiones, la resolución de los conflictos cotidianos, entre otros aspectos. Este currículo tiene que ser auténtico, es decir, que dé respuestas al contexto, para lo cual es necesaria una articulación entre ese entorno social-cultural, las características propias del niño, la niña, los objetivos y los contenidos de estudio, para poder desarrollarse lo más coherente que sea posible.

Gimeno (2000) afirma:

La educación tiene ideales y desempeña funciones muchas veces de carácter contradictorio, por pretender provocar la diferenciación individualizadora a la vez que la socialización homogeneizadora, que significa compartir rasgos de pensamiento, de comportamiento y de sentimiento con otros. Para hacer efectiva esta función socializadora, se ha utilizado la vía disciplinaria, la adhesión por convencimiento a normas y valores y la difusión de significados culturales. Seguramente, la pulsión socializadora por vía coercitiva y la proposición de modelos univocos de la cultura han predominado en la institución escolar sobre el valor de preservar y cultivar la individualidad. Aquí puede residir uno de los orígenes de que la diversidad siga siendo un problema y un reto (p. 16).

\section{Los procesos de aprendizaje y enseñanza de la lectura y la escritura des- de la atención de la diversidad para niños y niñas preescolares}

El proceso de aprendizaje, por su parte, implica que el alumnado desarrolle su individualidad, ya que es un proceso fundamentado en la actividad educativa que este desempeñe (Carrión y Sánchez, 2002, p. 204). Para ello es necesario, tal y como lo mencionan los autores antes citados, una columna organizativa basada en la "interacción, innovación y fiexibilidad" (p. 204); estos tres principios guiarán el proceso de enseñanza y aprendizaje.

La enseñanza es un espacio donde los sujetos mantienen un contacto interpersonal; ellos necesitan dialogar, sentir, experimentar, discutir, comentar, hablar, escuchar y expresar sus sentimientos y pensamientos. Por eso, es necesario el principio de la interacción, porque "sustenta la actividad, la participación y la cooperación como principios de enseñanza” (p. 205). 
Vigotsky (citado por Carrión y Sánchez, 2002), afirma que el lugar donde existe la interacción, será un aula con vida y diversidad.

La innovación puede desarrollarse mediante el cambio de la didáctica, la cual es definida como:

Una teoría de la enseñanza, heredada y deudora de muchas otras disciplinas. Su destino, empero, al ocuparse de la acción pedagógica es constituirse, reciprocamente, en oferente y dadora de teoría en el campo de la acción social y del conocimiento (Camilloni, 1997, p. 39).

Las docentes de la Educación Preescolar pueden recurrir a innovaciones, incorporar e innovar sus prácticas pedagógicas mediante el uso de los talleres, proyectos, rincones, trabajos, grupales; las tutorías entre iguales permiten que el o la estudiante preste su ayuda, desarrolle sus habilidades, se dé un aprendizaje recíproco. Esie tipo de actividades, como lo mencionan Carrión y Sánchez (2002), favorecen la atención a la diversidad, pues dan la oportunidad de desarrollar la enseñanza desde otra dimensión; estas altemativas metodológicas desarrollan cualquier área del currículo y el alumno y la alumna realizan diferentes actividades, rompiendo, así, la concepción de que todos deben hacer lo mismo.

Al hablar sobre enseñanza, aprendizaje y didáctica, es indispensable mencionar otro principio pedagógico, constituido por la concepción del modelo más acorde con la atención a la diversidad. Un modelo pedagógico "es la representación de las relaciones que predominan en el acto de enseñar, es también un paradigma que puede coexistir con otros y que sirve para organizar la búsqueda de nuevos conocimientos en el campo de la pedagogía" (Florez, 2000, p. 32).

Agrega el autor que un modelo pedagógico social-cognitivo permite que el individuo acceda a su propio desarrollo en el proceso de enseñanza y aprendizaje, de forma progresiva y secuencial con sus estructuras mentales; para ello el niño o la niña cuenta con un ambiente de experiencias, donde explora, investiga, construye hipótesis y las comprueba.

La experiencia vital del alumnado es muy importante en este enfoque, pero tiene una finalidad: contribuir al desarrollo, abrirse a experiencias superiores.

Los planteamientos teóricos hasta aquí señalados hacen ver la necesidad de poner la mirada en el aula preescolar; ahí la diversidad en los procesos 
de enseñanza y aprendizaje es latente, como es el caso de una atención a la diversidad en el proceso de la enseñanza y el aprendizaje de la lectura y la escritura. El niño y la niña construyen los esquemas comunicativos y el lenguaje lo es en su primera infancia, tal y como lo menciona Martínez (1997) mediante la "interacción con el medio físico" (p. 17).

La atención a la diversidad de los procesos de la lectura y escritura se puede llevar a cabo desde una forma temprana en el individuo, la cual promueve un inicio exitoso en el aprendizaje, pues de forma emergente, el niño y la niña demuestran actitudes de comprensión, funcionalidad del proceso comunicativo, en un entorno social definido. Tal y como lo menciona Ruiz (1996), en ese entorno, donde el niño y la niña aprenden mediante el encuentro natural y espontáneo con el lenguaje escrito, al utilizar estrategias como "claves semánticas, pruebas de hipótesis, y mediante la manipulación y exploración espontánea del lenguaje” (p. 11).

En el ambiente preescolar, es de gran importancia el valor que se le proporcione a la diversidad de los procesos de lectura y escritura, pues desde un enfoque de la teoría piagetiana y la Pedagogía Operatoria, el individuo es un protagonista. Sánchez, Flores y Céspedes (2001) enfatizan en la teoría de Piaget, ya que el sujeto en interacción con el objeto, realizará la construcción del conocimiento, mediante los procesos de asimilación y acomodación. Igualmente, la teoría afirma la importancia del espacio socioafectivo, porque se motiva la autonomía, las relaciones y resoluciones del conflicto. En conjunto, los cuatro principios cognitivos afirman que la enseñanza se debe dar en un entorno lúdico, donde se aprende del error para continuar, además de que el niño y la niña pueden explicar lo que hacen y, por último, se debe mantener como un proceso esa enseñanza.

Giroux (1990) hace una excelente visualización de lo que debería ser el proceso de la escritura en el siglo actual, al enfatizar en la revalorización que el pedagogo debe dar a la acción pedagógica que como tal cumple. Al propiciar la lucha constante por una sociedad más crítica, se busca el progreso de todos y el docente ha de ser capaz de dẻnunciar las ideologías que atentan contra los derechos humanos; además, para ello necesita inculcar también en sus estudiantes el sentido activo que cumplen en la sociedad.

\section{Descripción de los participantes}

En una institución educativa se puede ubicar una población bastante heterogénea, porque las y los estudiantes son diferentes por múltiples 
factores. Por ello, se introduce el concepto de diversidad. Meléndez (2002) define la escuela como

...centro donde confluyen y se representan los distintos caracteres de la heterogeneidad cultural y personal de la comunidad a la que pertenece. La escuela es el lugar exacto para aprovechar la riqueza de esa diversidad y para desarrollarse creyendo que todas las identidades valen; para promover una vida en democracia, de respeto mutuo y de cooperación solidaria; es el espacio justo para construir la paz y la equidad, enseñando a los estudiantes a responder ante la injusticia y la desigualdad (p. 33).

\section{Dimensiones de la diversidad en los sujetos participantes}

Los participantes de esta propuesta poseen sus propias características, que son visualizadas desde el enfoque de la diversidad, ya que presentan indicadores que los hacen realmente diversos:

\section{Participante 1:}

Fecha de nacimiento:

Edad:

Sexo:

Pais de origen:

Domicilio:

Nivel de preescolar que cursa:

Tiempo de asistir a la educación preescolar:

\section{Participante 2:}

Fecha de nacimiento:

Edad:

Sexo:

País de origen:

Domicilio:

Nivel de preescolar que cursa:

Tiempo de asistir a la educación preescolar:
05 de agosto de 1998

5 años y dos meses

Masculino

Costa Rica

Alajuelita

Kínder - Materno Infantil

8 meses actual

21 de diciembre de 1997

5 años y 10 meses

Femenino

Costa Rica

Aserrí

Kínder - Materno Infantil

6 meses $1^{\circ}$ Centro Educ. 4 meses $2^{\circ}$ Centro Educ.

8 meses actual 


\section{Participante 3:}

Fecha de nacimiento:

Edad:

Sexo:

País de origen:

Domicilio

Nivel de preescolar que cursa:

Tiempo de asistir a la educación preescolar:
25 de noviembre de 1997

5 años y 10 meses

Femenino

México

Escazú

Kínder - Materno Infantil 1 año $1^{\circ}$ Centro Educ.

8 meses actual

\section{Participante 4:}

Fecha de nacimiento:

19 de abril de 1998

Edad:

Sexo:

País de origen:

Domicilio

Nivel de preescolar que cursa:

Tiempo de asistir a la educación preescolar:

5 años y 6 meses

Femenino

Costa Rica

Guachipelín, Escazú

Kínder - Materno Infantil

1 año $1^{\circ}$ Centro Educ.
8 meses actual

\section{Participante 5:}

Fecha de nacimiento:

Edad:

Sexo:

País de origen:

Domicilio:

Nivel de preescolar que cursa:

Tiempo de asistir a la educación preescolar:

\section{Participante 6:}

Fecha de nacimiento:

Edad:

Sexo:

País de origen:

Domicilio:

Nivel de preescolar que cursa:

Tiempo de asistir a la educación preescolar:
06 de noviembre de 1997 5 años y 10 meses

Masculino

Costa Rica

Guachipelín, Escazú

Kínder - Materno Infantil

8 meses actual

28 de julio de 1998

5 años y 2 meses

Femenino

Costa Rica

Bello Horizonte, Escazú

Kínder - Materno Infantil

1 año $1^{\circ}$ Centro Educ.

8 meses actual 


\section{Participante 7:}

Fecha de nacimiento:

11 de enero de 1998

Edad:

5 años y 9 meses

Sexo:

Femenino

País de origen:

Costa Rica

Domicilio:

Alajuelita

Nivel de preescolar que cursa:

Kinder - Materno Infantil

Tiempo de asistir a la educación preescolar:

8 meses actual

\section{Participante 8:}

Fecha de nacimiento:

16 de setiembre de 1998

Edad:

5 años y 1 mes

Sexo:

Masculino

País de origen:

Costa Rica

Domicilio:

San Antonio, Escazú

Nivel de preescolar que cursa:

Kínder - Materno Infantil

Tiempo de asistir a la educación preescolar: 8 meses actual

\section{Participante 9:}

Fecha de nacimiento:

10 de julio de 1998

Edad:

5 años y 3 meses

Sexo:

Femenino

País de origen:

Costa Rica

Domicilio:

Santa Ana

Nivel de preescolar que cursa:

Kínder - Materno Infantil

Tiempo de asistir a la educación preescolar: 8 meses actual

\section{Participante 10:}

Fecha de nacimiento:

16 de julio de 1998

Edad:

5 años y 2 meses

Sexo:

País de origen:

Masculino

Domicilio:

Nivel de preescolar que cursa:

Costa Rica

Bebedero, Escazú

Kínder - Materno Infantil

Tiempo de asistir a la educación preescolar: 


\section{Caracterización general de la diversidad de los participantes:}

La diversidad de género: Se identifican 6 niñas y 4 niños en el diagnóstico, quienes poseen las características propias de sus identidades.

La diversidad personal o individual: Es contemplado con la edad de cada uno de los participantes, la cual es determinante en la madurez escolar y el desenvolvimiento en las actividades educativas; la edad oscila entre 5 años y tres meses y 6 años y seis meses. Al conocer la edad de cada uno de los participantes, se puede comprender su desarrollo en el contexto escolar $y$, por ende, los avances que logran en los procesos de lectura y escritura.

El diagnóstico indicó los niveles de preescolar que han cursado los niños y las niñas. Este lo denomina el Ministerio de Educación Pública, como Materno Infantil. En comparación con otros centros infantiles, el centro en estudio tiene sus diferencias; la más marcada es el aspecto de educación privada que posee y a partir de ello, se desligan otros aspectos, como los perfiles, fines y objetivos de la institución.

En los sujetos participantes, son notables sus diferencias. Un aspecto de la diversidad de la historia escolar es el tiempo de asistir a la Educación Preescolar; en este sentido, las y los participantes han recibido diferente tiempo la estimulación de un centro educativo, o bien, en el contexto familiar; ello hace diferente el proceso de enseñanza y aprendizaje, porque al tener mayor acceso a la estimulación temprana, el discente tiene mejores motivaciones en su desarrollo integral, además de que cada uno posee su propio bagaje de conocimientos previos que utilizan para enfrentar los conflictos cognitivos. Por ende, la diversidad personal es vivenciada desde una perspectiva que contempla al ser humano como integral y con sus propias concepciones y formas de desarrollarse en el ambiente escolar.

La diversidad por estilos de aprendizaje: Es otro aspecto que generó el diagnóstico, y tiene mucha relación con el anterior, ya que cada uno de los discentes, al ser totalmente diferente al otro, posee sus propias características en el momento del proceso de enseñanza y aprendizaje. El funcionamiento en el aula demuestra las diferentes formas en cómo los participantes se desarrollan en el contexto de aula. Hay quienes, por ejemplo, sienten deseos de participar en todo tipo de actividades y, por el contrario, existen niños y niñas a los que no les gustan las actividades grupales.

La diversidad es la cultura: Todos los niños y las niñas provienen de diferentes sectores, que poseen sus propias características. Por ejemplo, el cantón de Escazú es sumamente desarrollado en el ámbito económico, ya que cuenta con diversas actividades comerciales. Prácticamente, la zona 
rural ha ido desapareciendo para dar paso a infraestructuras comerciales y de recreación, para un sector de la población, con los índices de acceso con una economía de tipo media o media alta y alta. La participante que proviene de la zona de Aserrí, se desarrolla en un contexto que aún conserva un poco más el carácter de pueblo. Por su parte, Guachipelín también está enfrentando la llegada de la globalización, un poco más retardada que en el centro de Escazú. El cantón de Alajuelita presenta zonas urbanas marginales y existen mayores índices de violencia y de robos.

Para el estudio realizado, las manifestaciones de la diversidad fueron registradas en el proceso del diagnóstico, utilizando instrumentos elaborados con la finalidad de recolectar datos identificadores de la diversidad. Se elaboró un instrumento que permitió recolectar toda la información de identificación de los participantes del proceso investigativo. Otro instrumento recogió la información referente a la diversidad de la historia escolar del discente. La información del Contexto Familiar y Socioeconómico del niño y la niña, se recopiló mediante un instrumento que permitió conocer aspectos del núcleo familiar.

\section{Propósitos de la propuesta}

La aceptación de la diversidad en el aula plantea para el o la docente un reto durante toda su práctica profesional. Desde esta óptica, la lectura y la escritura, en el caso particular desde este estudio, son también un ámbito que desafia al docente. Si la diversidad es la que marca la dinámica del aula, entonces, la lectura y la escritura se constituyen en un proceso que ha de responder a esa diversidad, para que realmente se atiendan en el aula las diferencias.

La atención a la diversidad del desarrollo de la lectura y la escritura, específicamente en el Ciclo Preescolar, implica una valorización de los factores intrapersonales como la personalidad, ritmo y estilos de aprendizaje, las capacidades cognitivas, además de los factores interpersonales que inciden en aspectos como la cultura, las características de formación del docente y el contexto familiar y social.

Entonces, surgen las controversias al respecto del aprendizaje de la lectura y la escritura. Las incógnitas expresan: ¿cuándo es el momento apto para iniciar el proceso?, ¿es necesario utilizar un método?, ¿cuál es el idioma más apto para iniciar el proceso, en caso de escuelas bilingües?, ¿cómo desarrollar el proceso de la lectura y escritura cuando los padres del infante poseen un nivel socioeducativo que no posibilita un ambiente estimulante? 
Esta propuesta tiene como elementos fundamentales los siguientes aspectos para un abordaje de los procesos de la lectura y la escritura en el aula preescolar:

El aprendizaje es individual, el desarrollo mantiene una autonomía, por lo tanto, el ser humano debe saber cómo aprende y cuáles son sus habilidades y destrezas más evolucionadas.

No todos los niños y las niñas aprenden de forma igual, porque su diversidad los determina; se habla de las inteligencias múltiples y algunos desarrollan las habilidades visuales, auditivas, de movimiento, interpersonales, intrapersonales, que influyen en el desarrollo de los procesos de lectura y escritura en niños y niñas preescolares. Cada ser humano se desarrolla de diversas formas, dependiendo del contexto, la estructura familiar, los estímulos del ambiente escolar, familiar y social, lo cual fomenta la formación de un ser humano único e irrepetible.

La mediación es fundamental en el proceso de la lectura y la escritura; la o el docente debe mantener una actitud de disponibilidad, aceptación de las individualidades, además de un espíritu de creatividad para mejorar cada día su intervención pedagógica.

\section{Propósitos específicos de la propuesta}

1. Desarrollar el nivel del lenguaje oral en todos los y las estudiantes mediante estrategias donde la diversidad de cada uno sea la que marque el inicio del proceso y se mantenga durante su desarrollo:

1.1 Estimular la habilidad de hablar y escuchar, lo que cada uno piensa y concibe como importante para luego valorarla y aceptarla.

1.2 Lograr un correcto desarrollo fonológico, manteniendo la idea de que son diferentes en toda su forma de pronunciar.

1.3 Identificar las estructuras previas en el aspecto morfosintáctico, considerando las diferencias que pueden suscitarse durante el proceso.

2. Ajustar las estrategias a la edad cronológica y maduracional, respetando la diferencia que representa cada uno de los párvulos:

2.1 Diagnosticar la edad cronológica y maduracional del párvulo, respetando la diversidad que generan los resultados.

2.2 Elaborar las estrategias acordes con los conocimientos previos, los intereses y las necesidades del niño y la niña.

3. Desarrollar la discriminación auditiva y fonética de acuerdo con las capacidades de cada estudiante, respetando sus diferencias. 
4. Fomentar el lenguaje creativo en diversas actividades, donde la originalidad sea un principio invariable.

5. Dar un acercamiento a la estructura convencional de los códigos del lenguaje escrito, partiendo de la diversidad en los niños y las niñas.

6. Favorecer el desarrollo de habilidades metalingüísticas.

\section{Contenidos de la propuesta}

La realidad que se vivencia en los centros infantiles en torno a los procesos de la lectura y la escritura es muy diversas. Por lo tanto, esta propuesta tiene como áreas de interés:

1. El lenguaje oral en los niños y las niñas preescolares partiendo de su diversidad.

1.1 La habilidad de hablar y escuchar a quienes lo rodean y la aceptación de criterios diferentes.

1.2 Habilidades fonológicas diversas en los compañeros y en él mismo o ella.

1.3 Habilidades morfosintáctico de acuerdo con la diversidad del niño y la niña.

2. Diversidad en la edad cronológica y maduracional.

2.1 La diversidad en el centro infantil: edades de los párvulos y los procesos de la lectura y la escritura desde una visión de diversidad.

2.2 Estimulación del proceso de lectura y la escritura de acuerdo con el individuo, sus conocimientos previos en las destrezas y habilidades de la lectoescritura.

3. Discriminación auditiva y fonética.

4. Desarrollo de la creatividad a partir del abordaje de los procesos de lectura y la escritura.

5. La estructura convencional de los códigos del lenguaje escrito a partir de diversos métodos, respetando las diferencias de los individuos.

6. Habilidades metalingüísticas.

\section{Estrategias de mediación}

La estrategia didáctica que se utiliza para la consecución de los propósitos, se basa en los talleres para el desarrollo de los procesos de lectura y escritura para la diversidad, los cuales se especifican seguidamente: 
TALLER No 1: "Soy un ser humano único que puedo comunicarme con las personas que me rodean". Autora: Silvia Elena León Muñoz.

Objetivo general: Desarrollar el lenguaje oral en la niño y el niña como medio de comunicación de los intereses, necesidades y deseos que poseen.

Descripción: Con este taller, se busca que la o el estudiante exprese las características que lo identifican como una persona única, ante diversas personas de sus contextos: familia, institución y aula. En el entorno familia pueden generarse conversaciones donde se destaquen sus gustos y a la vez, pueden utilizar el dibujo como parte de la experiencia, y así compartirlo en el contexto del aula. Pretende desarrollar el sentido de la escucha, el valor de la diversidad personal y el lenguaje oral.

A partir de la exposición, se ejecutan actividades de articulación o dicción para desarrollar las habilidades fonéticas de más dificultad en los niños y las niñas: ejercicios de entonación, ejercicios de respiración, ejercicios de trabalenguas, donde se inculca en el niño y la niña el respeto por quienes lo hacen de forma diferente.

TALLER N0 2: "Aprender el valor de lo diferente a partir de hacer mis creaciones y conocer las de mis compañeros y compañeras para aprender el valor de lo diferente". Autora: Silvia Elena León Muñoz.

Objetivo: Diagnosticar el nivel de conceptualización de la lengua escrita en la que se encuentra el alumno o la alumna con la ubicación que hace Ruiz (1996), para conocer la diversidad del discente y partir de ella el proceso, tomando en cuenta además la edad cronológica y maduracional. También promover en el niño y la niña el valor de lo diferente, como un aspecto de importancia en la convivencia con las personas que lo rodean.

Descripción: En el aula, la y el estudiante pueden construir cartas, rótulos, diarios, tarjetas, un distintivo para él o ella y sus pertenencias de acuerdo con los diversos intereses y habilidades utilizando diferentes materiales. Se busca mantener su personalidad en el estilo y la forma de construir sus proyectos, además de aceptar lo que los otros realizan. La docente mantiene su función de facilitadora del proceso.

Los discentes pueden disfrutan de leer diferentes textos según sus posibilidades y manteniendo el respeto por quienes lo hacen de forma diferente, sin segregar a quienes no lo pueden llevar a cabo. A partir de la lectura, se pueden generar espacios de conversación y dramatización de las diferentes historias creadas por cada estudiante, tratando de respetar las ideas de los demás y las propias, de manera que la vivencia de la diversidad se visualice en todo momento. 
TALLER No 3: "Las maravillas que puedo escuchar". Autora: Silvia Elena León Muñoz.

Objetivo: Fomentar la habilidad de la escucha y reproducción de diferentes elementos (canciones, narraciones, entre otras) para desarrollar las habilidades auditivas y fonéticas, respetando el ser único de los otros.

Descripción: Con este taller se busca desarrollar la audición: sonidos básicos para el desarrollo de la lectura y la escritura, que pueden ser presentados como grabaciones, las cuales se pueden adivinar, o bien para reproducir. Se logrará una expresión de la diversidad que cada uno de los y las estudiantes. Se puede incentivar al y la estudiante a crear centros de grabaciones y de escucha de discos o casetes donde cada participante parte de su diverso estilo personal.

TALLER No 4: "Me gusta crear mis tarjetas, canciones, poesías, cartas y conocer las creaciones de mis compañeros y compañeras para convivir en diversidad". Autora: Silvia Elena León Muñoz.

Objetivo: Despertar el interés por la lectura y la escritura y propiciar en el niño y la niña la comprensión de la lectura y la escritura para valorarla y gozarla, partiendo de los diversos niveles de desarrollo y promover el trabajo en equipo.

Descripción: En este taller el uso del dibujo en los y las estudiantes, facilita la creación de libros de historia, entre otros productos. La y el estudiante utilizan cualquier forma de lenguaje, sin segregar a quienes no lo hacen de la misma manera, para favorecer la enseñanza cooperativa. Por lo tanto, la diversidad es el punto de partida y la aceptación de los productos de sus semejantes es la meta a alcanzar. 
TALLER No 5: "Las letras y palabras son parte de mi vida y me agrada conocerlas", Autora: Silvia Elena León Muñoz.

Objetivo: Crear ambientes llenos de la lengua escrita para que los niños y las niñas puedan observarlas, conocerlas e incorporarlas a sus proyectos.

Descripción: Para este taller es importante que la y el estudiante propongan las actividades que les gustan y responden a su diversidad personal, con el objetivo de plantear, por ejemplo, Talleres de Lectura Creativa.

Se debe también incorporar en el aula un Centro de Lectura y Escritura para el uso del discente, partiendo de sus diversos intereses y necesidades, las cuales serán respetadas por todos los integrantes del nivel.

La lectura a los compañeros y las compañeras tanto de forma individual como grupal de diferentes historias, puede responder a la diversidad que él o ella poseen, con esto se logra una vivencia de aceptación de sus habilidades y las de los demás.

\section{Instrumental evaluativo}

Los talleres poseen la evaluación particular para cada uno, así como una serie de metas para lograr el objetivo.

La evaluación general de la propuesta consiste en una lista de cotejo, que a su vez se subdivide en dos apartados, en el primero se enfatiza en función del desarrollo de los objetivos de la lectura y la escritura. En el segundo, se valorizan las dimensiones de la diversidad en la aplicación de la propuesta pedagógica. Este instrumental evaluativo se aplicará una vez concluidos los talleres, para así aportar las conclusiones correspondientes. 
Aguilar, L. (2000). De la integración a la inclusividad. Buenos Aires: Espacio Editorial.

Abramovich, M. (s.f.). Lamento por los niños del mundo.

Abramovich, M. (s.f.). La chinita y la rosa.

Barrantes, R. (2000). Investigación: un camino al conocimiento, un enfoque cuantitativo y cualitativo. 1 era. edición. San José: Editorial de la Universidad Estatal a Distancia.

Bravo, L. (2004) www.reduc.ci Revista digital UMBRAL 200 N$^{\circ} 14-$ Enero 2004. Camilloni, A. (1997). Corrientes didácticas contemporáneas. Argentina: Paidós.

Cantillano, K., Rodríguez, S. y Ruiz, S. (1996). Lenguaje Integral: Una propuesta curricular para el nivel de preescolar. Tesis presentada a la División de Educación Básica, Centro de Investigación y Docencia en Educación, de la Universidad Nacional para optar por el grado de Licenciatura en Ciencias de la Educación con énfasis en Educación Preescolar.

Carrión, J., Sánchez, P. (2002). Educación especial. Centros educativos y profesores ante la diversidad. España: Ediciones Pirámide.

Cerdas, V., Cerdas, W., Díaz, L., Solano, P. (2000). Filosofia Lenguaje Integral. Sistematización de algunas experiencias planteadas en el modelo pedagógico de la Escuela Nueva Laboratorio de la Universidad de Costa Rica. Tesis de Licenciatura no publicada. Universidad Nacional, Heredia.

Cid, J. (1997). Los pobres cuentan. San José: FLACSO.

Condemarín, M. (1999). Lectura temprana (Jardin Infantil y Primer Grado). Chile: Editorial Andrés Bello.

Danoff, J., Breitbart, V. y Bar, E. (1996). Iniciación con los niños. Para quienes se interesan en la educación de los niños de edad temprana. ( $4^{\text {a }}$. reimp.) México: Editorial Trillas.

Devalle de Rendo, A. y Vega, V. (1999). Una escuela en y para la diversidad. Sao Paulo: Aique Grupo Editor S.A.

Ferreiro, E. y Teberosky, A. (1979). Los sistemas de escritura en el desarrollo del niño. México: Siglo XX.

Florez, R. (2000). Evaluación pedagógica y cognitiva. Colombia: Mc Graw Hill. Gimeno, J. (2000). Atención a la diversidad. España: Editorial Graó.

Giroux (1990) Los profesores como intelectuales. Hacia una pedagogia crítica del aprendizaje. España: Ediciones Piados.

Gómez, M. (1982). "La adquisición de la lengua escrita". En: Resumen del capitulo IV. Propuesta para el Aprendizaje de la Lengua escrita. México, D.F.: SEDOEA. 
Goodman, K. (1989). Lenguaje Integral. Venezuela: Editorial Venezolana C.A.

Hemández. H. (s.f.). Un árbol de navidad.

Kamofsky, F., Weiss, T. (1993). Cómo aumentar la destreza de su hijo para el lenguaje y el pensamiento. Estados Unidos: Fearon Teacher Aids.

Martínez, M. (1997). Los procesos de la lectura y la escritura. Colombia: Editorial Universidad del Valle.

Meléndez, L. (2002). La inclusión escolar del alumno con discapacidad intelectual. Colombia: GLARP-IIPD/IIP-UCR y MEP- C.R.

Moreno, M. (s.f.). El globo dormilón.

Moreno, M. y el equipo del IMIPAE (1986). La pedagogía operatoria. Un enfoque constructivista de la educación. Barcelona: España. Editorial Laia.

Pfister, M. (s.f.). El pez arco iris.

Ramírez, P., Ramírez, C. (2001). Propuesta metodológica para la mediación pedagógica en los procesos iniciales de lectura y escritura para niños de 5 y 6 años que asisten a un jardin de infantes privados. Tesis presentada en la División de Educación Básica, Centro de Investigación y Docencia en Educación, de la Universidad Nacional, para optar el grado de Licenciatura en Ciencias en Educación con énfasis en Educación Preescolar.

Ruiz, D. (1996). La alfabetización temprana en el ambiente preescolar. Nuevas tendencias para aprender a leer y escribir. Puerto Rico: Editorial First Book Publishing of P.R.

Sánchez, M., Flores, L., Céspedes, E. (2001). Re-pensar la práctica pedagógica. Costa Rica: Ministerio de Educación Pública, OIMM USAID, Universidad Nacional. 